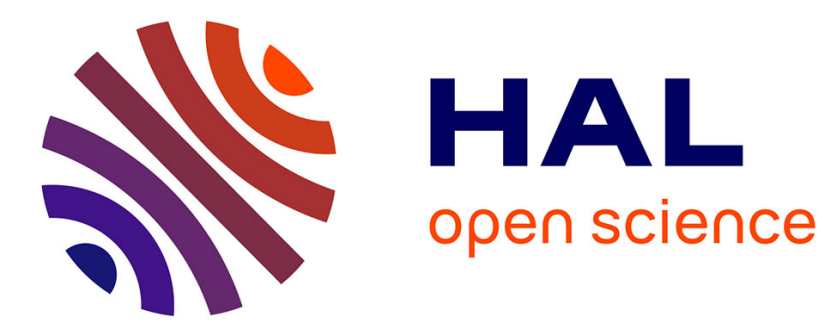

\title{
Epsilon: A Library of Software Tools for Polynomial Elimination
}

Dongming Wang

\section{To cite this version:}

Dongming Wang. Epsilon: A Library of Software Tools for Polynomial Elimination. Proceedings of the First International Congress of Mathematical Software - ICMS 2002, Aug 2002, Pékin, Chine, pp.379-389. inria-00107607

\section{HAL Id: inria-00107607 https://hal.inria.fr/inria-00107607}

Submitted on 19 Oct 2006

HAL is a multi-disciplinary open access archive for the deposit and dissemination of scientific research documents, whether they are published or not. The documents may come from teaching and research institutions in France or abroad, or from public or private research centers.
L'archive ouverte pluridisciplinaire HAL, est destinée au dépôt et à la diffusion de documents scientifiques de niveau recherche, publiés ou non, émanant des établissements d'enseignement et de recherche français ou étrangers, des laboratoires publics ou privés. 


\title{
EPSILON: A LIBRARY OF SOFTWARE TOOLS FOR POLYNOMIAL ELIMINATION
}

\author{
DONGMING WANG \\ LIP6, UPMC-CNRS, 4 place Jussieu, 75252 Paris Cedex 05, France \\ E-mail: Dongming.Wang@lip6.fr.http://calfor.lip6.fr/ rwang
}

\begin{abstract}
This article presents a Maple library of functions for decomposing systems of multivariate polynomials into triangular systems of various kinds (regular, simple, or irreducible), with an application package for manipulating and proving geometric theorems.
\end{abstract}

\section{Introduction}

It has been widely recognized that zero-decomposition algorithms based on triangular sets are efficient tools for polynomial elimination and computation. They are ideal alternatives to algorithms based on Gröbner bases and resultants for many geometric applications. The popularization of triangular-set-based methods suffers from the lack of available implementations, while in striking contrast Gröbner basis packages may be found in almost all computer algebra systems. A recent effort to improve the situation is made by Aubry and Moreno Maza ${ }^{1}$ who have implemented the four methods of Wu, Lazard, Kalkbrener, and the author in Axiom. This comparative implementation has also been integrated into Aldor by Moreno Maza. Another earlier implementation of Wu's method, carried out by Gao and Chou, has been included in the system $\mathrm{GEX}^{3}$ for automated geometric reasoning.

The author has been advocating the development of triangular set methods for more than a decade. One of his efforts is the maintenance of the CharSets package, ${ }^{8}$ which provides a general and complete implementation of $\mathrm{Wu}$-Ritt's method of characteristic sets, ${ }^{5,14,15}$ together with a few relevant algorithms, in Maple. It seems that this package still represents one of the most comprehensive and widely available implementations of the characteristic set method. Part of the package was translated by M. W. Messollen into a library for Singular and Macaulay 2 and by T. Shimoyama into Risa/Asir for the purpose of primary decomposition. ${ }^{6}$ It is being translated by D. Stanger into Maxima.

In 1993 the author proposed a method for decomposing any system of multivariate polynomials into triangular systems, with or without projection, and into irreducible triangular systems. ${ }^{7}$ This method makes use of some elimination strategies from Wu-Ritt's method and Seidenderg's theory and is practically more efficient. In 1998 and 2000, the author proposed another method, based on the computation of regular subresultants, for decomposing any polynomial system into simple systems ${ }^{11}$ and regular systems. ${ }^{12}$ Meanwhile, we have implemented these methods as two sepa- 
rate packages, named TriSys and SiSys, also in Maple for research experiments. The Epsilon library described in this paper consists of CharSets, TriSys, SiSys, and another application package GEOTHER ${ }^{10}$ (for geometric theorem proving). We present the implementation, functionality, performance, and availability of Epsilon, together with some practical information for the user.

Besides the above-mentioned packages, there exist several implementations of triangular set algorithms, in particular, algorithms based on Wu-Ritt's characteristic sets. $^{2}$ However, most of these implementations have quite limited availability. The reader is referred to the books $\mathrm{s}^{3,13,16}$ for remarkable applications of triangular set methods and software tools in geometric reasoning.

\section{Design and Implementation of Epsilon}

Epsilon is a library of functions implemented in Maple mostly and in Java for polynomial elimination with applications. Some of the Java routines for geometric drawing were written previously in C. This library has evolved from the author's continuous effort to put elimination theory and methods into practice. The functions available in the current version of the library are capable of performing a number of computational tasks including:

- Decomposing polynomial systems into triangular systems of various kinds;

- Decomposing algebraic varieties into unmixed or irreducible subvarieties, and polynomial ideals into primary components;

- Factorizing polynomials over successive algebraic extension fields;

- Solving systems of polynomial equations and inequations;

- Manipulating and proving theorems in elementary and differential geometry automatically.

There are also functions which can decompose any system of ordinary differential polynomials into (irreducible) differential triangular systems. Main capabilities and the dependence of the four Epsilon modules are summarized in Fig. 1.

The implementation of Epsilon started with the CharSets module in the fall of 1989. The first version CharSets 1.0 running with Maple 4.3 was ready for public distribution in later 1990. This self-contained package implements a collection of fundamental algorithms, including two little known algorithms for polynomial factorization over algebraic extension fields, based on the computation of Wu-Ritt's characteristic sets. ${ }^{5,14,15}$

Along with the implementation of CharSets, we created an environment GEOTHER for manipulating and proving theorems in geometry. With the powerful algo-

wang: submitted to World Scientific on April 28, 2002 
rithms from CharSets and TriSys as its algebraic engine, GEOTHER provides a set of functions for the manipulation, translation, diagram generation, and automated proving of geometric theorems. An early version of GEOTHER was ready for demonstration in 1991, and in 1996 was published a short description of the enhanced version. ${ }^{10}$

CharSets is an independent toolkit, whereas all the other modules need routines from CharSets and thus depend on CharSets. Some of the functions in CharSets, TriSys, and SiSys do the same tasks but with different performances (see Fig. 3). A distinct feature of SiSys lies in its capability of computing regular systems and simple systems, while TriSys' functions for zero decomposition remain the most efficient. The four packages were designed and implemented at different times along with the author's algorithmic research. Most of the algorithms implemented in Epsilon are described formally in the book. ${ }^{13}$ Some of them have similar functionalities, but they differ in terms of structure and computational efficiency.

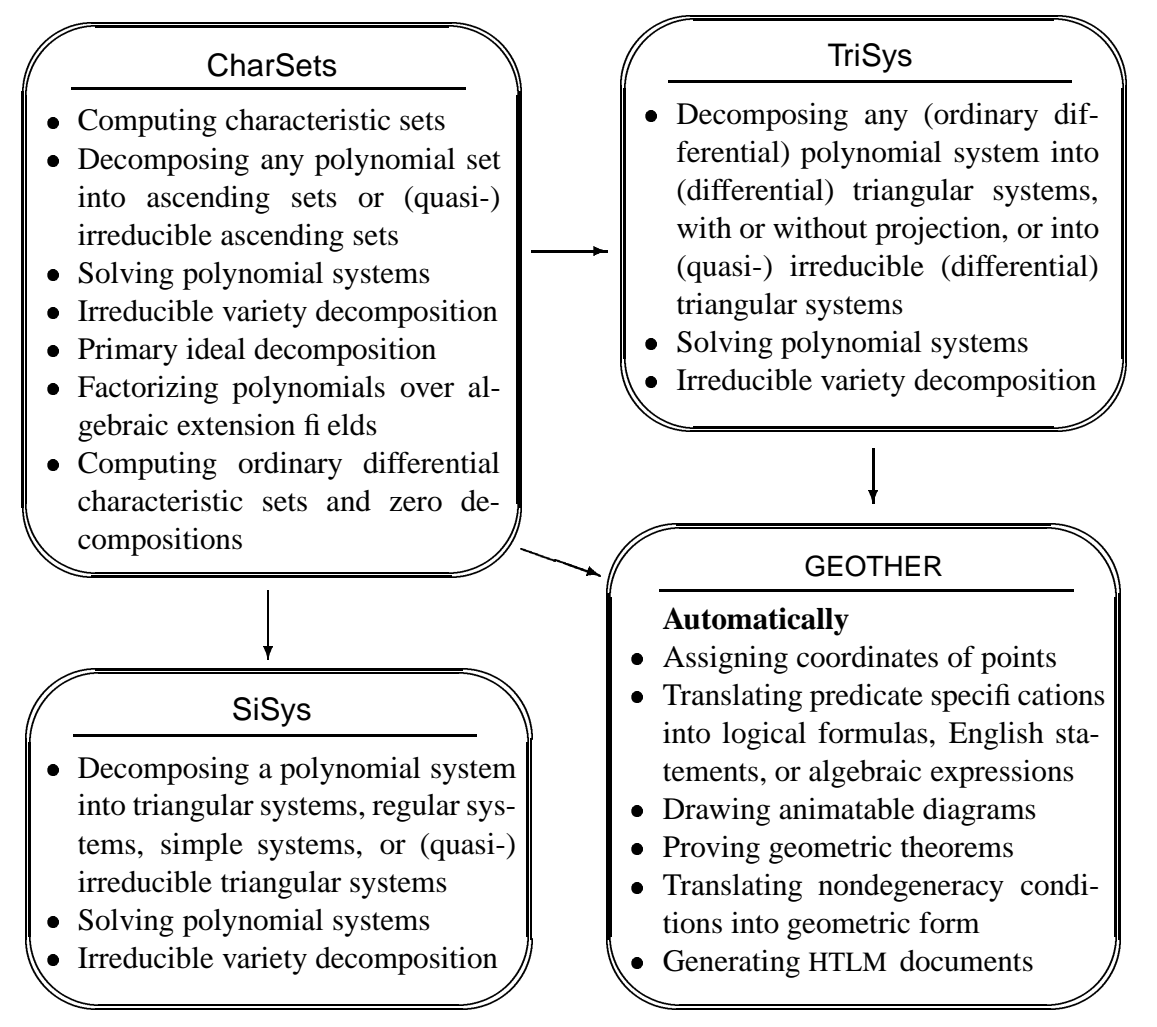

Figure 1. Functionality and relationship of Epsilon packages 
Maple was chosen as the basis of our packages for its easy-to-use high-level programming language, its potential and availability, and most importantly, its variety and efficiency of basic functions on polynomial operation and elimination. The flourishing development and increasing popularity of Maple as a general-purpose computer algebra system have proven the soundness of our early choice.

In brief, Epsilon was not designed a priori; it has been developed progressively by incorporating up-to-date research accomplishments. It is a unique and rich library for triangular-set-based polynomial elimination. Its functions for zero decomposition are all Gröbner bases free (see relevant remarks by Gräbe ${ }^{4}$ ). More technical and implementational details for each Epsilon package will be discussed elsewhere.

\section{Epsilon Functions}

The Epsilon functions are chosen from the large number of functions in CharSets, TriSys, SiSys, and GEOTHER to help the user perform common computations without entering into each package. These functions are either often used or the most efficient in general (according to our experiments and experience). We present some of them in this section.

All polynomials mentioned below are in the variables $x_{1}, \ldots, x_{n}$ with coefficients in $\mathcal{K}$, the field $Q$ of rational numbers or the ring $Q\left[u_{1}, \ldots, u_{d}\right]$ with $u_{i}$ considered as parametric variables. A polynomial set is a finite set of nonzero polynomials, and a polynomial system $[\mathbb{P}, \mathbb{Q}]$ is a pair of polynomial sets. Define

$$
\operatorname{Zero}(\mathbb{P} / \mathbb{Q}):=\left\{\begin{array}{l|l}
\left({ }^{-} x, \ldots,{ }^{-x}\right) \in \overline{\mathcal{K}}^{n} & \begin{array}{l}
P\left({ }^{-} x, \ldots,{ }^{-} x\right)=0, \forall P \in \mathbb{P}, \\
Q\left({ }^{-} x, \ldots,-{ }^{-}\right) \neq 0, \forall Q \in \mathbb{Q}
\end{array}
\end{array}\right\},
$$

where $\overline{\mathcal{K}}$ is an algebraically closed field containing $\mathcal{K}$. We shall write Zero $(\mathbb{P})$ for $\operatorname{Zero}(\mathbb{P} / \mathbb{Q})$ when $\mathbb{Q} \subset \mathcal{K} \backslash\{0\}$.

Let $P$ be any nonzero polynomial. The class of $P$, denoted by $\operatorname{cls}(P)$, is the biggest index of the variables $x_{k}$ that effectively occur in $P$ if $P \notin \mathcal{K}$, and 0 otherwise. Let $p=\operatorname{cls}(P)>0$; we call the leading coefficient of $P$ with respect to $x_{p}$ the initial of $P$, denoted by ini $(P)$.

\subsection{Characteristic Sets: CharSet}

Inputted a list $\mathrm{X}=\left[x_{1}, \ldots, x_{n}\right]$ of variables and a set $\mathrm{P}$ of polynomials in $\mathrm{X}$, CharSet $(\mathrm{P}, \mathrm{X})$ returns an $\mathbb{F}$-modified quasi-characteristic set $\mathbb{C}$ of $\mathrm{P}$ with respect to the variable ordering $x_{1} \prec \cdots \prec x_{n}$. For example, 


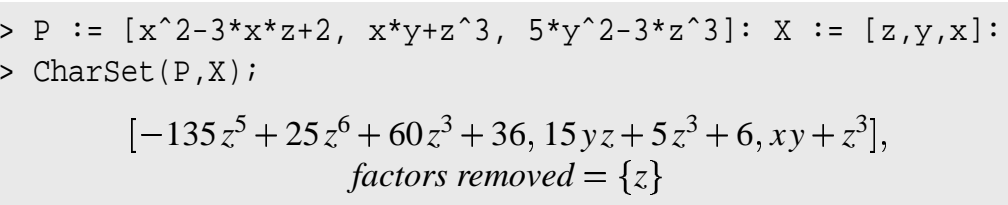

where $\mathbb{C}$ is the list (ordered set) of the three polynomials and $\mathbb{F}=\{z\}$, the set of removed factors.

Definition $1 \mathrm{~A}$ finite nonempty ordered set $\mathbb{T}=\left[T_{1}, T_{2}, \ldots, T_{r}\right]$ of polynomials is called a triangular set if $0<\operatorname{cls}\left(T_{1}\right)<\operatorname{cls}\left(T_{2}\right)<\cdots<\operatorname{cls}\left(T_{r}\right)$. A quasi-ascending set is either a triangular set or a set of a single nonzero polynomial of class 0 .

A quasi-ascending set $\mathbb{C}$ is called an $\mathbb{F}$-modified quasi-characteristic set of a polynomial set $\mathbb{P}$ if Zero $(\mathbb{P} / \mathbb{F}) \subset \operatorname{Zero}(\mathbb{C})$ and $\operatorname{remset}(\mathbb{P}, \mathbb{C})=\varnothing$, where $\mathbb{F}$ is another polynomial set.

Here the set of nonzero pseudo-remainders of a polynomial set $\mathbb{P}$ with respect to an arbitrary quasi-ascending set $\mathbb{A}=\left[A_{1}, \ldots, A_{r}\right]$, with $\operatorname{cls}\left(A_{i}\right)=p_{i}$, is defined as

$$
\begin{aligned}
& \operatorname{remset}(\mathbb{P}, \mathbb{A}):=\{\operatorname{prem}(P, \mathbb{A}) \mid P \in \mathbb{P}\} \backslash\{0\}, \quad \text { where } \\
& \operatorname{prem}(P, \mathbb{A}):= \begin{cases}0 & \text { if } r=1 \text { and } p_{1}=0, \\
\operatorname{prem}\left(\ldots \operatorname{prem}\left(P, A_{r}, x_{p_{r}}\right), \ldots, A_{1}, x_{p_{1}}\right) & \text { otherwise, }\end{cases}
\end{aligned}
$$

and prem $(P, A, x)$ denotes the pseudo-remainder of $P$ with respect to $A$ in $x$.

\subsection{Triangular Series: TriSer}

Inputted a list $\mathrm{X}=\left[x_{1}, \ldots, x_{n}\right]$ of variables and a polynomial system $[\mathrm{P}, \mathrm{Q}]$ in $\mathrm{X}$, $\operatorname{TriSer}([\mathrm{P}, Q], \mathrm{X})$ returns a fine triangular series $\left[\mathbb{T}_{1}, \mathbb{U}_{1}\right], \ldots,\left[\mathbb{T}_{e}, \mathbb{U}_{e}\right]$ of $[\mathrm{P}, Q]$ under the variable ordering $x_{1} \prec \cdots \prec x_{n}$.

Definition 2 A polynomial system $[\mathbb{T}, \mathbb{U}]$ is called a triangular system if $\mathbb{T}$ is a triangular set and $I\left({ }^{-} x, \ldots,{ }^{-} \mathfrak{x}\right) \neq 0$ for any $I \in\{\operatorname{ini}(T) \mid T \in \mathbb{T}\}$ of class $k$ and $\left({ }^{-} x, \ldots,{ }^{-} x\right) \in \operatorname{Zero}\left(\mathbb{T} \cap \mathcal{K}\left[x_{1}, \ldots, x_{k}\right] / \mathbb{U}\right)$. A triangular system $[\mathbb{T}, \mathbb{U}]$ is said to be fine if $\operatorname{prem}(U, \mathbb{T}) \neq 0$ for all $U \in \mathbb{U}$.

A sequence of (fine) triangular systems $\left[\mathbb{T}_{1}, \mathbb{U}_{1}\right], \ldots,\left[\mathbb{T}_{e}, \mathbb{U}_{e}\right]$ is called a (fine) triangular series of a polynomial system $[\mathbb{P}, \mathbb{Q}]$ if

$$
\operatorname{Zero}(\mathbb{P} / \mathbb{Q})=\bigcup_{i=1}^{e} \operatorname{Zero}\left(\mathbb{T}_{i} / \mathbb{U}_{i}\right)
$$

\subsection{Solving Polynomial Systems: TSolve}

Inputted a set $\mathrm{X}=\left\{x_{1}, \ldots, x_{n}\right\}$ of variables and a polynomial system $[\mathrm{P}, \mathrm{Q}]$ in $\mathrm{X}$, TSolve $([P, Q], X)$ returns the isolated solutions of $P=0, Q \neq 0$, and a sequence of 
fine triangular systems $\left[\mathbb{T}_{1}, \mathbb{U}_{1}\right], \ldots,\left[\mathbb{T}_{e}, \mathbb{U}_{e}\right]$ such that $\bigcup_{i=1}^{e} \operatorname{Zero}\left(\mathbb{T}_{i} / \mathbb{U}_{i}\right)$ represents the set of all the positive-dimensional solutions of $P=0, Q \neq 0$.

Note that $\mathbb{P}=0, \mathbb{Q} \neq 0$ mean the system of polynomial equations $P=0$ and inequations $Q \neq 0$ for all $P \in \mathbb{P}$ and $Q \in \mathbb{Q}$. In the books ${ }^{13,16}$ one may find different properties of regular, simple, and irreducible triangular systems introduced below.

\subsection{Regular Series: RegSer}

Inputted a list $\mathrm{X}=\left[x_{1}, \ldots, x_{n}\right]$ of variables and a polynomial system $[\mathrm{P}, \mathrm{Q}]$ in $\mathrm{X}$, RegSer $([\mathrm{P}, \mathrm{Q}], \mathrm{X})$ returns a regular series $\left[\mathbb{T}_{1}, \mathbb{U}_{1}\right], \ldots,\left[\mathbb{T}_{e}, \mathbb{U}_{e}\right]$ of $[\mathrm{P}, \mathrm{Q}]$ under the variable ordering $x_{1} \prec \cdots \prec x_{n}$.

Definition 3 A triangular system $[\mathbb{T}, \mathbb{U}]$ is called a regular system if (a) $\operatorname{cls}(T) \neq$ $\operatorname{cls}(U)$ for all $T \in \mathbb{T}$ and $U \in \mathbb{U}$; (b) $\operatorname{ini}(U)\left({ }^{-} x, \ldots,{ }^{-}{ }^{-}-1\right) \neq 0$ for any $1 \leq k \leq n$, $U \in \mathbb{U}$ of class $k$, and

$$
\left({ }^{-}, \ldots,{ }^{-}{ }^{-1}\right) \in \operatorname{Zero}\left(\mathbb{T} \cap \mathcal{K}\left[x_{1}, \ldots, x_{k-1}\right] / \mathbb{U} \cap \mathcal{K}\left[x_{1}, \ldots, x_{k-1}\right]\right) .
$$

A sequence of regular systems $\left[\mathbb{T}_{1}, \mathbb{U}_{1}\right], \ldots,\left[\mathbb{T}_{e}, \mathbb{U}_{e}\right]$ is called a regular series of a polynomial system $[\mathbb{P}, \mathbb{Q}]$ if (1) holds.

\subsection{Simple Series: Simser}

Inputted a list $\mathrm{X}=\left[x_{1}, \ldots, x_{n}\right]$ of variables and a polynomial system $[\mathrm{P}, \mathrm{Q}]$ in $\mathrm{X}$, SimSer $([\mathrm{P}, \mathrm{Q}], \mathrm{X})$ returns a simple series $\left[\mathbb{T}_{1}, \tilde{\mathbb{T}}_{1}\right], \ldots,\left[\mathbb{T}_{e}, \tilde{\mathbb{T}}_{e}\right]$ of $[\mathrm{P}, Q]$ with respect to the ordering $x_{1} \prec \cdots \prec x_{n}$.

Definition 4 A pair $[\mathbb{T}, \tilde{\mathbb{T}}]$, in which $\mathbb{T}$ and $\tilde{\mathbb{T}}$ are either triangular sets or the empty set, is called a simple system if (a) $\mathbb{T} \cap \tilde{\mathbb{T}}=\varnothing$ and $\mathbb{T} \cup \tilde{\mathbb{T}}$ can be reordered as a triangular set; (b) for every $T \in \mathbb{T} \cup \tilde{\mathbb{T}}$ of class $k$ and any

$$
\left({ }^{-} x, \ldots,{ }^{-} x_{-1}\right) \in \operatorname{Zero}\left(\mathbb{T} \cap \mathcal{K}\left[x_{1}, \ldots, x_{k-1}\right] / \tilde{\mathbb{T}} \cap \mathcal{K}\left[x_{1}, \ldots, x_{k-1}\right]\right),
$$

$\operatorname{ini}(T)\left({ }^{-} x, \ldots,{ }^{-}{ }^{-1}\right) \neq 0$ and $T\left({ }^{-} x, \ldots,{ }^{-}{ }^{-1}, x_{k}\right)$ is squarefree.

A sequence of simple systems $\left[\mathbb{T}_{1}, \mathbb{U}_{1}\right], \ldots,\left[\mathbb{T}_{e}, \mathbb{U}_{e}\right]$ is called a simple series of a polynomial system $[\mathbb{P}, \mathbb{Q}]$ if (1) holds.

\subsection{Irreducible Characteristic Series: ICS}

Inputted a list $\mathrm{X}=\left[x_{1}, \ldots, x_{n}\right]$ of variables and a polynomial system $[\mathrm{P}, \mathrm{Q}]$ in $\mathrm{X}$, $\operatorname{ICS}([\mathrm{P}, \mathrm{Q}], \mathrm{X})$ returns an irreducible characteristic series $\left[\mathbb{T}_{1}, \mathbb{U}_{1}\right], \ldots,\left[\mathbb{T}_{e}, \mathbb{U}_{e}\right]$ of $[\mathrm{P}, \mathrm{Q}]$ with respect to the ordering $x_{1} \prec \cdots \prec x_{n}$. 
Definition 5 A fine triangular set $\mathbb{T}=\left[T_{1}, \ldots, T_{r}\right]$ is said to be irreducible if there do not exist any integer $k(1 \leq k \leq n)$ and polynomials $D, F, G$ with $\operatorname{cls}(D)<$ $\operatorname{cls}(F)=\operatorname{cls}(G)=\operatorname{cls}\left(T_{k}\right)$ such that $D T_{k}=F G$ (when $\left.k=1\right)$ or prem $\left(D T_{k}-\right.$ $\left.F G,\left[T_{1}, \ldots, T_{k-1}\right]\right)=0$.

A fine triangular series $\left[\mathbb{T}_{1}, \mathbb{U}_{1}\right], \ldots,\left[\mathbb{T}_{e}, \mathbb{U}_{e}\right]$ of a polynomial system $[\mathbb{P}, \mathbb{Q}]$ is called an irreducible characteristic series of $[\mathbb{P}, \mathbb{Q}]$ if $\mathbb{T}_{i}$ is irreducible for each $i$.

\subsection{Irreducible Variety Decomposition: IVD}

Inputted a set $\mathrm{X}=\left\{x_{1}, \ldots, x_{n}\right\}$ of variables and a polynomial set $\mathrm{P}$ in $\mathrm{X}, \operatorname{IVD}(\mathrm{P}, \mathrm{X})$ returns a sequence of irredundant polynomial sets $\mathbb{P}_{1}, \ldots, \mathbb{P}_{e}$ such that

$$
\operatorname{Zero}(\mathrm{P})=\bigcup_{i=1}^{e} \operatorname{Zero}\left(\mathbb{P}_{i}\right)
$$

and each $\operatorname{Zero}\left(\mathbb{P}_{i}\right)$ as an algebraic variety is irreducible.

We do not define the standard notions of irreducible algebraic variety and primary ideal (see the references ${ }^{6,13,16}$ ).

\subsection{Primary Ideal Decomposition: PID}

Inputted a set $\mathrm{X}=\left\{x_{1}, \ldots, x_{n}\right\}$ of variables and a polynomial set $\mathrm{P}$ in $\mathrm{X}, \mathrm{PID}(\mathrm{P}, \mathrm{X})$ returns a sequence of irredundant polynomial sets $\mathbb{P}_{1}, \ldots, \mathbb{P}_{e}$ such that

$$
\operatorname{Ideal}(\mathrm{P})=\bigcap_{i=1}^{e} \operatorname{Ideal}\left(\mathbb{P}_{i}\right)
$$

and each $\operatorname{Ideal}\left(\mathbb{P}_{i}\right)$ is a primary ideal, where $\operatorname{Ideal}(\mathbb{P})$ denotes the ideal generated by all the polynomials in $\mathbb{P}$.

\subsection{Differential Triangular Series: dTriser}

Inputted a list $\mathrm{X}=\left[t, x_{1}, \ldots, x_{n}\right]$ of variables and an ordinary differential polynomial system $[\mathrm{P}, \mathrm{Q}]$ in $\mathrm{X}$ and $\mathrm{d}^{k} x_{i} / \mathrm{d} t^{k}, \operatorname{dTriSer}([\mathrm{P}, \mathrm{Q}], \mathrm{X})$ returns a differential triangular series $\left[\mathbb{T}_{1}, \mathbb{U}_{1}\right], \ldots,\left[\mathbb{T}_{e}, \mathbb{U}_{e}\right]$ of $[\mathrm{P}, \varrho]$ under the variable ordering $x_{1} \prec \cdots \prec x_{n}$.

Definition 6 A sequence of differential triangular systems $\left[\mathbb{T}_{1}, \mathbb{U}_{1}\right], \ldots,\left[\mathbb{T}_{e}, \mathbb{U}_{e}\right]$ is called a differential triangular series of a differential polynomial system $[\mathbb{P}, \mathbb{Q}]$ if $(1)$ holds, when zeros of polynomials are replaced by differential zeros of differential polynomials. 


\subsection{Proving Geometric Theorems: Prove}

Inputted the specification $\mathrm{T}$ of a geometric theorem, Prove( $\mathrm{T}$ ) proves or disproves $\mathrm{T}$, with subsidiary conditions provided.

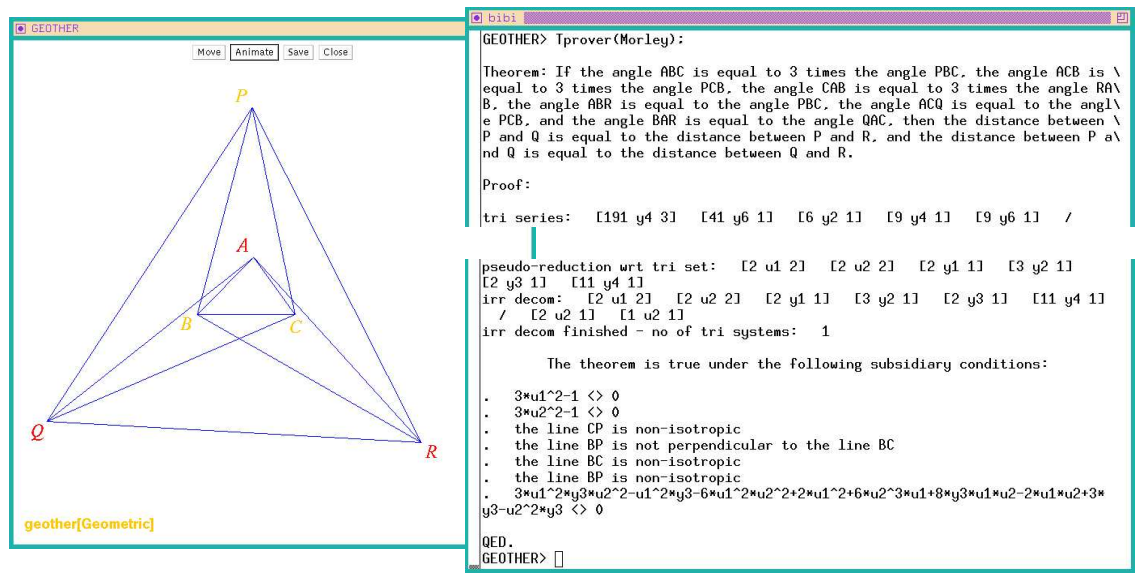

Figure 2. A GEOTHER session

Figure 2 reproduces a proof session and a diagram drawn automatically by GEOTHER for Morley's trisector theorem.

\section{Experiments}

Experiments ${ }^{7,9,1}$ show that the decomposition functions in TriSys are more efficient than those in CharSets in most cases. Table 1 is provided not for comparison but to give the reader an impression about the performance and applicability of Epsilon functions. The computations were done in Maple V.5 on an i686 under Linux 2.2.12 for some of the 50 test examples from the references, ${ }^{7,9}$ and $\infty$ means " $>3600$."

Consider, for instance, the decomposition of a polynomial set into irreducible triangular sets, which can be done by the function trisys [its]=ICS, sisys [its], or charsets [ics] from TriSys, SiSys, or CharSets. Figure 3 illustrates the performances of these functions in terms of computing time for some other examples.

\begin{tabular}{|c|rrrrrrrrrrr}
\hline Example & 1 & 2 & 5 & 12 & 14 & 16 & 17 & 19 & 21 & 22 & 24 \\
\hline TriSer & 1.3 & 73.9 & .8 & 2.7 & 123 & 290 & 8.3 & .6 & $\infty$ & 1.5 & 1.1 \\
RegSer & 2.4 & 28.7 & 4.8 & 1.4 & $\infty$ & 8.2 & 280 & 7 & $\infty$ & 22.8 & 7 \\
SimSer & 6.1 & 45.2 & 5.6 & 2 & $\infty$ & 7.2 & $\infty$ & 8.2 & $\infty$ & 18 & 28.5 \\
ICS & 3.4 & 86 & 1.2 & .6 & 378 & 135 & 19.4 & 1.2 & 4.7 & 3 & 3.4 \\
IVD & 7 & 100 & 1.2 & .8 & 392 & 149 & 19.6 & 1.2 & 5.6 & 3.4 & 4 \\
\hline
\end{tabular}




\begin{tabular}{rrrrrrrrrrrr|}
\hline 26 & 29 & 30 & 32 & 33 & 35 & 39 & 41 & 43 & 44 & 47 & 49 \\
\hline .2 & .1 & .6 & .3 & 6.1 & 3.4 & 3 & 1357 & 2.7 & 18.8 & .8 & 6.3 \\
.8 & 2 & 88 & 1 & 8.9 & 9.3 & 10.8 & 33 & 966 & 13.5 & 13.6 & $\infty$ \\
.9 & 2.6 & 89 & $\infty$ & $\infty$ & 10.5 & 16.6 & 44.6 & 1197 & $\infty$ & $\infty$ & 7501 \\
14.6 & 1.5 & 1.4 & .5 & 6.5 & 4.6 & 4.5 & 581 & 17.9 & 5.7 & 12.7 & 31.2 \\
16.4 & 1.6 & 1.4 & .5 & 4.8 & 5 & 4.6 & 571 & 17.5 & 12.9 & $\infty$ & 35.1 \\
\hline
\end{tabular}

Table 1. Computing times (CPU secs) for five kinds of decompositions

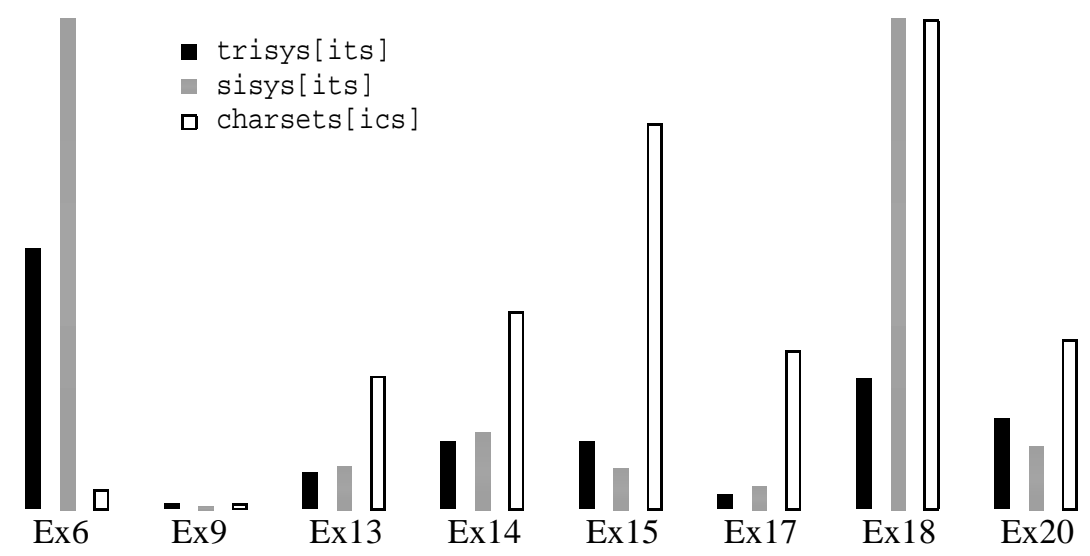

Figure 3. Timings for irreducible decomposition

\section{Interface, Availability, and Documentation}

\subsection{Interface}

More than $80 \%$ of Epsilon code has been written as Maple programs. This includes the entirety of code for the three modules CharSets, TriSys, SiSys, and the algebraic-computation part of GEOTHER. Actually, Epsilon is nothing else but a user-contributed Maple library and its modules can be used as standard Maple packages. Therefore, Epsilon functions can be defined and called either in command-line Maple or in X-Maple with graphic interface.

However, some of the functions in GEOTHER need external programs written in Java and interact with the operating system. These functions might not work under certain operating systems and Java installations. This concerns in particular the automatic generation of diagrams and HTML documentation for geometric theorems.

The Maple function system is simply used for the interaction with the operating system. The pipe facility of Unix and Linux operating systems is used for the im- 
plementation of a graphic interface, by means of which the user can call GEOTHER functions with mouse click.

\subsection{Availability}

The CharSets package has been included in Maple's share library for distribution since 1991 and its latest version may be downloaded from http://calfor.lip6.fr $/ \sim$ wang/charsets. The entire Epsilon library version 0.618 will be distributed with a book entitled "Elimination Practice: Software Tools and Applications." The author will continue enhancing the library, and its future versions released will be made available on the author's web page. Since the library is distributed with Maple source code, most of its functions may be used on any platform on which the corresponding version of Maple is installed.

\subsection{Documentation}

The first part of the above-mentioned book will serve as a general introduction to the Epsilon library. In Maple, the library, its modules, and all the user functions have online help with examples. The help facility has been created and may be used in the usual way as standard Maple packages and functions. In the CD included with the book the reader will find an HTML documentation of Epsilon with many examples. Revised and improved versions of the document will also be made available on the author's web page.

Acknowledgments. The development of the Epsilon library has been supported by various funding sources during the author's stay at RISC-Linz and LIFIA/LEIBNIZIMAG, and currently by the SPACES project (http://www.spaces-soft.org) and the Chinese national 973 project NKBRSF G19980306.

\section{References}

1. Aubry, P., Moreno Maza, M. (1999): Triangular sets for solving polynomial systems: A comparative implementation of four methods. J. Symb. Comput. 28: $125-54$.

2. Chou, S.-C., Gao, X.-S., Liu, Z., Wang, D.-K., Wang, D. (2000): Geometric theorem provers and algebraic equation solvers. In: Mathematics Mechanization and Applications (X.-S. Gao and D. Wang, eds.), pp. 491-505. Academic Press, London.

3. Gao, X.-S., Zhang, J.-Z., Chou, S.-C. (1998): Geometry Expert (in Chinese). Nine Chapters Publ., Taiwan. 
4. Gräbe, H.-G. (1999): About the polynomial system solve facility of Axiom, Macsyma, Maple, Mathematica, MuPAD, and Reduce. In: Computer Algebra Systems: A Practical Guide (M. Wester, ed.), pp. 121-151. Wiley, Chichester.

5. Ritt, J. F. (1950): Differential Algebra. Amer. Math. Soc., New York.

6. Shimoyama, T., Yokoyama, K. (1996): Localization and primary decomposition of polynomial ideals. J. Symb. Comput. 22: 247-277.

7. Wang, D. (1993): An elimination method for polynomial systems. J. Symb. Comput. 16: 83-114.

8. Wang, D. (1995): An implementation of the characteristic set method in Maple. In: Automated Practical Reasoning: Algebraic Approaches (J. Pfalzgraf and D. Wang, eds.), pp. 187-201. Springer, Wien New York.

9. Wang, D. (1996): Solving polynomial equations: Characteristic sets and triangular systems. Math. Comput. Simul. 42: 339-351.

10. Wang, D. (1996): GEOTHER: A geometry theorem prover. In: Automated Deduction (M. A. McRobbie and J. K. Slaney, eds.), LNAI 1104, pp. 166-170. Springer, Berlin Heidelberg.

11. Wang, D. (1998): Decomposing polynomial systems into simple systems. $J$. Symb. Comput. 25: 295-314.

12. Wang, D. (2000): Computing triangular systems and regular systems. J. Symb. Comput. 30: 221-236.

13. Wang, D. (2001): Elimination Methods. Springer, Wien New York.

14. Wu, W.-t. (1984): Basic principles of mechanical theorem proving in elementary geometries. J. Syst. Sci. Math. Sci. 4: 207-235.

15. Wu, W.-t. (1987): A zero structure theorem for polynomial-equations-solving. Math. Mech. Res. Preprints 1: 2-12.

16. Wu, W.-t. (1994): Mechanical Theorem Proving in Geometries: Basic Principles. Springer, Wien New York. 